\title{
Heparin-binding EGF-like growth factor protects intestinal stem cells from injury in a rat model of necrotizing enterocolitis
}

\author{
Chun-Liang Chen, Xiaoyi Yu, lyore O-A James, Hong-yi Zhang, Jingyuan Yang, Andrei Radulescu, \\ Yu Zhou and Gail E Besner
}

Necrotizing enterocolitis (NEC) is an often catastrophic disease that typically affects premature newborns. Although the exact etiology of NEC is uncertain, the disease is associated with formula feeding, bacterial colonization of the gut, hypoxia and hypoperfusion. In light of the pathogenesis of NEC, the integrity and function of the intestinal mucosa has a major defensive role against the initiation of NEC. Various forms of intestinal injury, including NEC, injure the intestinal epithelial cell (IEC) lineages, including the intestinal stem cells (ISCs), thereby disrupting the normal homeostasis needed to maintain gut barrier function. In the current study, we examined the effects of heparin-binding EGF-like growth factor (HB-EGF) administration on enterocytes, goblet cells, neuroendocrine cells and ISCs in a newborn rat model of experimental NEC. We also examined the cytoprotective effects of HB-EGF on ISCs in in vitro cell cultures and in ex vivo crypt-villous organoid cultures. We found that HB-EGF protects all IEC lineages, including ISCs, from injury. We further found that HB-EGF protects isolated ISCs from hypoxic injury in vitro, and promotes ISC activation and survival, and the expansion of crypt transit-amplifying cells, in ex vivo crypt-villous organoid cultures. The protective effects of HB-EGF were dependent on EGF receptor activation, and were mediated via the MEK $1 / 2$ and PI3K signaling pathways. These results show that the intestinal cytoprotective effects of HB-EGF are mediated, at least in part, through its ability to protect ISCs from injury.

Laboratory Investigation (2012) 92, 331-344; doi:10.1038/labinvest.2011.167; published online 12 December 2011

KEYWORDS: epithelial cells; HB-EGF; intestine; LGR5; prominin-1; stem cells

Necrotizing enterocolitis (NEC) is an often catastrophic disease that is the most common surgical emergency in the neonatal intensive care unit, and which most commonly affects premature infants weighing $<1.5 \mathrm{~kg}$. $^{1}$ Although the exact etiology of NEC is uncertain, the specific pathologic hallmarks of NEC include inflammatory cell infiltration, mucosal edema, ulceration and coagulative necrosis. ${ }^{2}$ An initial insult resulting in early epithelial injury induces release of inflammatory mediators that further cause compromised gut barrier function. Subsequent bacterial translocation and toxin absorption amplify the inflammatory cascade, worsening the epithelial injury. Extensive barrier failure and ensuing intestinal tissue necrosis are the ultimate manifestations of NEC. ${ }^{1}$ In light of the pathogenesis of NEC, the integrity and function of the intestinal mucosa has a major defensive role against the initiation of NEC.
The integrity of the intestinal epithelium is ensured by pluripotent, self-renewing and proliferative stem cells. ${ }^{3,4}$ These cells have only recently been identified using special markers such as Leucine-rich repeat-containing G-protein coupled receptor 5 (LGR5) and prominin-1/CD133, in addition to classic +4 long retention cell characteristics. ${ }^{5,6}$ Between 4 and 6 stem cells at each crypt base generate epithelial progenitor cells in the transit-amplifying (TA) zone, which subsequently differentiate and maintain intestinal homeostasis. ${ }^{3,4}$ They provide a fast-paced renewal of the four differentiated epithelial cell lineages, each of which has distinct important physiologic functions: enterocytes that absorb nutrients, goblet cells that produce protective mucus, Paneth cells that secrete antibacterial proteins and neuroendocrine cells that produce hormones. ${ }^{7}$ Stresses such as intestinal ischemia can harm the intestinal epithelial cell (IEC)

Department of Pediatric Surgery, The Research Institute at Nationwide Children's Hospital, Center for Perinatal Research, Nationwide Children's Hospital, The Ohio State University College of Medicine, Columbus, $\mathrm{OH}$, USA

Correspondence: Dr GE Besner, MD, Department of Pediatric Surgery, The Research Institute at Nationwide Children's Hospital, Center for Perinatal Research, Nationwide Children's Hospital, The Ohio State University College of Medicine, ED321, 700 Children's Drive, Columbus, OH 43205, USA.

E-mail: gail.besner@nationwidechildrens.org

Received 6 June 2011; revised 14 September 2011; accepted 8 October 2011 
lineages, particularly the stem cells, thereby disrupting normal homeostasis and gut barrier function. Stem cells in some organs, including the intestines, have been shown to respond to stress and to promote recovery from injury. ${ }^{8}$ A previous study showed that bone marrow-derived progenitor cells have the ability to regenerate the intestine after injury. ${ }^{9}$ However, the role of intestinal stem cells (ISCs) in recovery from NEC is unknown. The ability to protect ISCs in the face of stress may represent a critical step in the prevention and treatment of NEC.

Previous studies from our laboratory have shown that heparin-binding EGF-like growth factor (HB-EGF) can protect the intestines from NEC. ${ }^{10,11}$ HB-EGF was first identified in the conditioned medium of cultured human macrophages, ${ }^{12}$ and was subsequently found to be a member of the EGF family. ${ }^{13}$ HB-EGF is initially produced as a 208 amino acid transmembrane precursor molecule (proHB-EGF) that undergoes extracellular proteolytic cleavage to yield the mature secreted form of the growth factor (sHB-EGF). ${ }^{14}$ In the intestine, we have shown that exogenous administration of HB-EGF promotes enterocyte migration, ${ }^{15}$ prevents IEC apoptosis, ${ }^{16}$ decreases bacterial translocation ${ }^{17}$ and preserves gut barrier function ${ }^{18}$ after injury. In addition, we showed that HB-EGF inhibits the expression of inflammatory cytokines, ${ }^{19}$ adhesion molecules and infiltration of inflammatory cells after intestinal injury. ${ }^{20}$ Although we have shown that enteral administration of HB-EGF promotes enterocyte proliferation in the face of intestinal injury, ${ }^{15}$ we have not investigated the effect of HB-EGF on ISCs or on the individual IEC lineages. In the current study, we examined the effects of HB-EGF administration on enterocytes, goblet cells, neuroendocrine cells and stem cells in a newborn rat model of experimental NEC. We further examined the effect of HB-EGF on isolated purified ISCs in vitro, and using a novel ex vivo crypt-villous organoid culture system.

\section{MATERIALS AND METHODS Rat Pup Model of Experimental NEC}

All experimental procedures were carried out according to guidelines for the ethical treatment of experimental animals and approved by the Institutional Animal Care and Use Committee of Nationwide Children's Hospital (Protocol \#04203AR). Experimental NEC was induced using a modification of the neonatal rat model of NEC initially described by Barlow and Santulli ${ }^{21}$ and modified as we have previously described. ${ }^{22}$ Rat pups were delivered on day 21 of gestation by Cesarean section under $\mathrm{CO}_{2}$ anesthesia from timed pregnant rats (Harlan Sprague-Dawley, Indianapolis, IN, USA). Newborn rat pups were maintained in an incubator at $37^{\circ} \mathrm{C}$ and gavage-fed with hypertonic formula containing $15 \mathrm{~g}$ Similac 60/40 (Ross Pediatrics, Columbus, OH, USA) in $75 \mathrm{ml}$ Esbilac (Pet-Ag, New Hampshire, IL, USA), a diet that provided $836.8 \mathrm{~kJ} / \mathrm{kg}$ daily. Feeds were started at $0.1 \mathrm{ml}$ every $4 \mathrm{~h}$ beginning $30 \mathrm{~min}$ after birth, and gradually increased to $0.4 \mathrm{ml}$ per feed. Pups $(n=10)$, designated as the NEC group, were exposed to hypoxia with $100 \%$ nitrogen for $1 \mathrm{~min}$, followed by hypothermia at $4{ }^{\circ} \mathrm{C}$ for $10 \mathrm{~min}$ twice daily beginning $60 \mathrm{~min}$ after birth for either 1,2 or 3 days, with intragastric feeding of lipopolysaccharide (LPS) $(2 \mathrm{mg} / \mathrm{kg}) 8 \mathrm{~h}$ after birth. LPS administration enhanced the incidence of NEC in our model and has been used by others as well. ${ }^{23}$ Pups were euthanized by cervical dislocation on the development of any clinical signs of NEC, or at the end of the experiment at 3 days after birth. Additional pups $(n=10)$, designated as the NEC + HB-EGF group, were stressed for 3 days, but were treated with HB-EGF ( $800 \mu \mathrm{g} / \mathrm{kg}$ per dose) added to each feed beginning with the first feed received after birth. Control pups $(n=5)$, designated as the breast milk (BM) group, were breast fed for 3 days using surrogate mothers (since their natural mothers were killed after C-section), and were not stressed. The recombinant human HB-EGF used in the current experiments, corresponding to amino acids $74-148$ of the mature HB-EGF precursor, was produced using a Pichia pastoris expression system according to Good Laboratory Practice procedures (Trillium Therapeutics, Toronto, Canada).

\section{Histological Injury Grading}

Intestines were removed on killing and fixed in 10\% formalin for $24 \mathrm{~h}$. Four pieces each of duodenum, jejunum, ileum and colon were harvested, paraffin-embedded, sectioned at $5 \mu \mathrm{m}$ thickness, and stained with hematoxylin and eosin. Intestinal injury was graded by examining tissue sections with phase contrast microscopy using the histological scoring system described by Caplan et al. ${ }^{24}$ Intestinal morphologic changes were graded as: grade 0 , no damage; grade 1, epithelial cell lifting or separation; grade 2, necrosis to the mid villous level; grade 3 , necrosis of the entire villus; and grade 4 , transmural necrosis. Histological injury scores of grade 2 or greater were considered positive for NEC. Grading was carried out blindly by two experienced independent observers (AR and XY).

Clinical NEC and atresia (control) samples were obtained from patients undergoing bowel resection in accordance with Nationwide Children's Hospital guidelines for human subjects research (IRB Approval \#06-00267). These tissues were processed in the same way as the rat pup intestinal samples.

\section{Histologic and Immunohistochemical Detection of IEC Lineages}

Rat pup jejunal cross-sections ( $5 \mu \mathrm{m}$ thickness) were subjected to histochemical and immunohistochemical staining for detection of IEC lineages.

\section{Enterocytes}

Enterocytes were identified by $\mathrm{H} \& \mathrm{E}$ staining of tissue sections. H\&E stained sections were examined using an Axioscope microscope (HBO 100/W2, Zeiss, Thornwood, NY, USA) with bright field photo-documentation using AxioVision software (version, 02.2002). Enterocytes in villi were manually identified and marked and then numerically 
counted using the Cell Counter in ImageJ software (version 1.39U, NIH, Bethesda, MD, USA).

\section{Goblet Cells}

Goblet cells were identified by periodic acid-Schiff (PAS) staining of tissue sections.

\section{Neuroendocrine Cells}

Immunofluorescent staining was performed for the detection of chromogranin-A-positive neuroendocrine cells using rabbit polyclonal anti-chromogranin-A $(\mathrm{v}: \mathrm{v}=1: 500)(\mathrm{ABCAM}$, Cambridge, MA, USA) primary antibodies.

\section{Paneth Cells}

As we were unable to identify granules characteristic of Paneth cells in the intervillous regions, tissue sections were also subjected to $\alpha$-defensin immunostaining using goat polyclonal anti- $\alpha$-defensin (R-19) (Santa Cruz Biotechnology, Santa Cruz, CA, USA) primary antibodies in an attempt to identify Paneth cells.

\section{ISCs and TA Cells}

Proliferating ISCs and TA cells were identified by immunostaining using mouse anti-proliferating cell nuclear antigen (PCNA) (Sigma-Aldrich, St Louis, MO, USA) primary antibodies, as previously described. ${ }^{25}$ ISCs were further identified by immunostaining using rabbit anti-LGR5 ( $(\mathrm{v}: \mathrm{v}=1: 500)$ (MBL International Corporation, Woburn, MA, USA $)^{5}$ and rat monoclonal anti-prominin-1 $(\mathrm{v}: \mathrm{v}=1: 10)$ (Miltenyi Biotec, Auburn, CA, USA). ${ }^{26,27}$

\section{Immunohistochemistry}

Tissue sections were rehydrated and then blocked with $10 \%$ donkey serum/PBS for $1 \mathrm{~h}$ at RT. Either rabbit polyclonal anti-chromogranin-A ( $\mathrm{v}: \mathrm{v}=1: 500)$, goat polyclonal anti- $\alpha$ defensin (R-19), mouse anti-PCNA, rabbit anti-LGR5 $(\mathrm{v}: \mathrm{v}=1: 500)$, rat monoclonal anti-prominin-1 $(\mathrm{v}: \mathrm{v}=1: 10)$ or rabbit anti-CD133 antibodies were applied to tissue sections according to the manufacturer's recommended dilutions in $10 \%$ donkey serum/PBS for $1 \mathrm{~h}$ at RT or overnight at $4{ }^{\circ} \mathrm{C}$ For double staining, two antibodies were added for incubation simultaneously. After three 10-min washes with PBS/0.1\% Tween 20, tissue sections were incubated with the appropriate anti-IgG $(4 \mu \mathrm{g} / \mathrm{ml})$ conjugated with FITC or Cy3 (Jackson ImmunoResearch Laboratories, West Grove, PA, USA) in $10 \%$ donkey serum/PBS for $1 \mathrm{~h}$ at RT. Tissue sections were mounted with ProLong Gold antifade reagent with DAPI (Invitrogen, Carlsbad, CA, USA) after three 10-min washes with PBS $+0.1 \%$ Tween 20 . Negative controls in the absence of primary antibodies were performed for all immunostaining to indicate the level of background staining intensity. The cells with immunostaining intensity above background staining were counted as positively staining cells. Fluorescence microscopy was performed using an AxioSkope fluorescent microscope (HBO 100/W 2, Zeiss).

\section{Quantification of IEC lineage Cells}

IEC lineage cells were quantified manually in 15 or more well-aligned villi demonstrating complete lymphatics.

\section{Isolation of prominin-1 ${ }^{+}$ISCs}

Magnetic-activated cell sorting (MACS) isolation of prominin-1 ${ }^{+}$ISCs was performed with modifications of a previously described method. ${ }^{28,29}$ Small intestines were excised from 6 to 10 neonatal rat pups at 3 days of age for isolation of intestinal progenitor and stem cells. Intestines were opened longitudinally, washed with cold PBS and cut into $5 \mathrm{~mm}$ pieces. Tissue fragments were incubated in $2 \mathrm{mM}$ EDTA/PBS for $30 \mathrm{~min}$ on ice. Intervillous epithelia were enriched and centrifuged at $150-200 \mathrm{~g}$ for $3 \mathrm{~min}$ as described previously, ${ }^{28}$ and dissociated by incubation in PBS supplemented with trypsin $(10 \mathrm{mg} / \mathrm{ml})$ and DNase $(0.8 \mu / \mu \mathrm{l})$ for $30 \mathrm{~min}$ at $37^{\circ} \mathrm{C} .{ }^{30}$ Single cells were centrifuged at $300 \mathrm{~g}$ for $10 \mathrm{~min}$ at $4{ }^{\circ} \mathrm{C}$, resuspended in minimum essential medium and filtered through $40 \mu \mathrm{m}$ cell strainers. Strained cells were washed with $10 \mathrm{ml}$ of cold PBS and centrifuged at $300 \mathrm{~g}$ for $10 \mathrm{~min}$ at $4{ }^{\circ} \mathrm{C}$. The isolation of prominin-1-positive stem cells was carried out according to the manufacturer's protocol (Miltenyi Biotec) as follows. Dissociated intervillous epithelial cells were resuspended in $80 \mu \mathrm{l} \mathrm{PBS} / \mathrm{BSA} / \mathrm{EDTA}$ buffer $(\mathrm{pH}$ 7.2, $0.5 \%$ BSA and $2 \mathrm{mM}$ EDTA) per $10^{7}$ total cells. Twenty $\mu \mathrm{l}$ of anti-Prominin-1 MicroBeads (Miltenyi Biotec) per $10^{7}$ total cells were added and incubated for $10 \mathrm{~min}$ on ice. Cells were washed with 1-2 $\mathrm{ml}$ of buffer per $10^{7}$ cells and centrifuged at $300 \mathrm{~g}$ for $10 \mathrm{~min}$. Supernatants were removed and $\sim 10^{8}$ cells were suspended in $500 \mu \mathrm{l}$ of PBS/BSA/EDTA buffer and run through MACS pre-separation filters to remove clumped cells. MACS separation columns were placed in a magnetic multistand and rinsed with $2 \mathrm{ml}$ PBS/BSA/EDTA buffer. Filtered cell suspensions were applied to the columns, the columns were washed two times with $2 \mathrm{ml}$ PBS/BSA/EDTA buffer, and flow through collected as controls. The retained prominin-1-positive cells were harvested by removing the column from the magnetic multistand, and eluting the cells into collection tubes using $2 \mathrm{ml}$ PBS/BSA/EDTA buffer. To monitor the purification efficiency, portions of run through and retained cells were centrifuged at $300 \mathrm{~g}$ at $4{ }^{\circ} \mathrm{C}$ and fixed in methanol/acetone $(\mathrm{v}: \mathrm{v}=1: 1)$ for $30 \mathrm{~min}$. After three washes with PBS buffer, cells were subjected to anti-prominin-1 antibody immunostaining. Prominin-1-positive stem cells were maintained in medium (high-glucose Dulbecco's modified Eagle's medium (DMEM) with 10\% FBS, $10 \mu \mathrm{g} / \mathrm{ml}$ insulin, $2 \mathrm{mM}$ glutamine, $100 \mathrm{U} / \mathrm{ml}$ penicillin and $100 \mu \mathrm{g} / \mathrm{ml}$ streptomycin) at $37^{\circ} \mathrm{C}$ in an incubator with $5 \% \mathrm{CO}_{2}$ until hypoxia experiments were carried out.

Additional experiments were designed to confirm that prominin-1 MACS enriches for ISC. MACS isolated cells were labeled either with anti-Prominin-1 and Cy3-conjugated secondary antibody or with anti-LGR5 and FITCconjugated secondary antibody, and then subjected to flow cytometry analysis (BD LSR II; BD Biosciences, San Jose, CA, 
USA) with 30000 events recorded. Appropriate controls were labeled with secondary antibodies conjugated with $\mathrm{Cy} 3$ or FITC alone.

\section{Ex Vivo Crypt-Villous Organoid Culture and Analysis Crypt Isolation}

These studies were approved by Institutional Animal Care and Use Committee of the Children's Research Institute (IACUC Protocol \# AR-06-00092). C57BL/6J 3-month-old mice were killed and the intestines removed. Crypt isolation was carried out using a modification of a previously described method. ${ }^{31}$ The distal half of the jejunum and the entire ileum were excised and intestinal contents were removed by flushing with ice-cold $\mathrm{Ca}^{2+}$ - and $\mathrm{Mg}^{2+}$-free PBS. The intestine was reverted on a $4 \mathrm{~mm}$ glass rod and exposed to PBS/EDTA ( $30 \mathrm{mM})(\mathrm{pH} 7.4)$, at $37^{\circ} \mathrm{C}$ for $5 \mathrm{~min}$. To release villi into ice-cold PBS, intestines on glass rods were assembled unto a Bulcher gradient maker and subjected to $4-5$ pulses of vibration. Sheets of crypts were then rapidly vibrated off the intestine into new ice-cold PBS after a further 15 -min incubation in PBS/EDTA ( $30 \mathrm{mM})(\mathrm{pH} 7.4)$, at $37^{\circ} \mathrm{C}$. Crypts were separated from remnant villi by gentle pipetting up and down with $10 \mathrm{ml}$ serum tubes followed by filtering through $70 \mu \mathrm{m}$ cell strainers. Crypts were centrifuged at $100-150 \mathrm{~g}$ and were resuspended in cold PBS buffer. Crypts were quantified using hemocytometry with Trypan blue (1:10 dilution) (Invitrogen).

\section{Ex Vivo Crypt-Villous Organoid Culture}

Crypt-villous organoid cultures were established according to the methodology described by Sato et al. ${ }^{28}$ The concentration of isolated crypts was evaluated by counting the total number of crypts in $100 \mu \mathrm{l}$ PBS microscopically. In all, $\sim 500$ crypts plus $50 \mu \mathrm{l}$ of BD Matrigel basement membrane matrix (BD Biosciences) were mixed and seeded in 24-well plates. When gels polymerized at $\mathrm{RT}, 500 \mu \mathrm{l}$ of crypt culture medium (advanced DMEM/F12) (Invitrogen) containing EGF (50 ng/ $\mathrm{ml}$ ) (Peprotech, Rocky Hill, NJ, USA) or HB-EGF (50 ng/ml) (Trillium Therapeutics), plus the Wnt agonist R-spondin 1 (500 ng/ml) (R\&D Systems, Minneapolis, MN, USA) and the BMP inhibitor Noggin $(100 \mathrm{ng} / \mathrm{ml})$ (Peprotech) were used to maintain crypt-villous organoid growth. In order to further examine the requirements for organoid growth, HB-EGF, R-spondin 1 or Noggin, alone or in various combinations, were added and replaced every three days. Crypt cultures were maintained at $37^{\circ} \mathrm{C}$ in an incubator with $5 \% \mathrm{CO}_{2}$ and the percentage of crypts growing into crypt-villous organoids were evaluated at days 1,3 and 5. Crypt-villous organoids were released from matrigel using recovery buffer (BD Biosciences) on ice for $30 \mathrm{~min}$ and washed in $1 \times$ PBS three times before fixation in $4 \%$ paraformaldehy/PBS for $2 \mathrm{~h}$. Orgnoids were penetrated using $0.1 \%$ Tween 20/PBS for immunostaining. Some organoids were embedded in histogel (Lab Storage System, St Peters, MO, USA) and fixed again in $10 \%$ formalin/PBS before paraffin embedding and section- ing. Organoid tissue sections were subjected to cell lineage identification using H\&E, immunohistologic and PAS staining as described above.

\section{Ex Vivo Crypt-Villous Organoid Analyses}

Ex vivo crypt-villous organoids were analyzed as follows. Crypt-villous organoid viability in each culture well was expressed as the percentage of viable organoids after scoring of at least 50 organoids. Organoid size was determined by microscopic visualization of 15 crypt-villous organoids at $\times 5$ magnification using a LEICA DM-4000B microscope, with organoid size expressed in relative area units obtained using ImageJ software (version 1.39U, NIH). Crypt length was quantified similarly and expressed as relative length units. The total number of crypts in each crypt-villous organoid was also determined. A relative unit is a pixel unit designated by ImageJ software when a certain length or area was measured.

\section{Exposure of Prominin-1 ${ }^{+}$ISCs and Ex Vivo Crypt-Villous Organoids to Hypoxia}

MACS-isolated prominin-1-positive cells $\left(10^{4}\right)$ were seeded in 96-well plates in triplicate and incubated overnight. Cells were subjected to hypoxia (100\% nitrogen) or to normoxia for $60 \mathrm{~min}$ in the presence or absence of HB-EGF $(100 \mathrm{ng} / \mathrm{ml})$ that was added $1 \mathrm{~h}$ before the initiation of hypoxia. Stem cell viability was evaluated 24-h post hypoxia using the Cyquant cell proliferation assay kit (Invitrogen, Eugene, OR, USA), normalized to the viability of the normoxic control without HB-EGF, which was designated as $100 \%$.

Ex vivo crypt-villous organoids were cultured overnight and subjected to hypoxia (100\% nitrogen) or to normoxia for $60 \mathrm{~min}$, in the presence or absence of HB-EGF $(50 \mathrm{ng} / \mathrm{ml})$ that was added $12 \mathrm{~h}$ before hypoxia. Each treatment was performed in triplicate. Crypt viability in 50 crypts was examined on days 1-5 after hypoxia, with determination of the percentage of crypts that formed crypt-villous organoids. The size of crypt-villous organoids exposed to different treatments at days $1-5$ of culture was normalized to the size of crypt-villous organoids exposed to normoxia for 1 day.

\section{Inhibition of HB-EGF Signaling}

To examine the signaling pathways used by HB-EGF for promoting stem cell survival and growth of crypt-villous organoids, crypts were cultured in the presence of HB-EGF $(50 \mathrm{ng} / \mathrm{ml})$ in addition to R-spondin 1 and noggin. The EGFR inhibitor AG1478 ${ }^{32}(1.5 \mu \mathrm{M}$; Calbiochem, Gibbsontown, NJ, USA), the PI3K inhibitor Ly294002 $2^{33}(60 \mu \mathrm{M}$; Calbiochem $)$ or the MEK1/2 inhibitor PD $98059^{34}(60 \mu \mathrm{M}$; Calbiochem) were added $30 \mathrm{~min}$ before the addition of HB-EGF. After $24 \mathrm{~h}$, the $\%$ of proliferative crypts was quantified. Results were normalized to the $\%$ of proliferative crypts grown in the absence of the inhibitors. 


\section{Statistical Analyses}

Data are presented as mean \pm s.e.m. from at least three independent experiments. Statistical analyses were performed using one-way, two-way and three-way ANOVA, with either Tukey-Kramer or Newman-Keuls pair-wise comparison tests, using SAS software (Version 92, SAS). $P<0.05$ was considered statistically significant.

\section{RESULTS}

\section{Administration of HB-EGF Protects Enterocytes, Goblet} Cells and Neuroendocrine Cells from NEC In Vivo

We found that HB-EGF protects enterocytes, goblet cells and neuroendocrine cells from injury because of experimental NEC in vivo (Figures 1a and b). Enterocytes/villous in breast fed control rat pups (BM group) were decreased significantly in pups with experimental NEC (NEC group), and increased significantly in pups with experimental NEC that were treated with HB-EGF added to the feeds (NEC + HB-EGF group). Similar results were found for goblet cells and neuroendocrine cells. No Paneth cells were detectable in the intervillous regions of newborn rat pups using either H\&E staining or anti- $\alpha$-defensin immunostaining (data not shown).

\section{HB-EGF Protects Rat Pup Intestinal Progenitor Cells and Stem Cells from NEC In Vivo}

PCNA immunostaining was used to identify proliferating ISCs and TA progenitor cells in the intervillous regions of rat pup intestines (Figure 2a). The PCNA antibodies labeled most of the intervillous epithelial cell nuclei in breast fed rat pups, indicating intense proliferation of these cells. PCNA immunostaining was markedly reduced in pups subjected to NEC. Importantly, pups subjected to NEC but treated with HB-EGF added to the feeds had significantly increased intervillous PCNA immunostaining compared with non-HBEGF-treated pups. These findings show that HB-EGF is able to protect stem cells/TA progenitor cells from experimental NEC.

Along with others, we have shown that LGR5 and prominin- 1 are both expressed in ISCs. ${ }^{5,6,26,27,35}$ To examine the effects of HB-EGF on ISCs specifically, we utilized LGR5 and prominin-1 immunostaining. Under basal, non-injury a
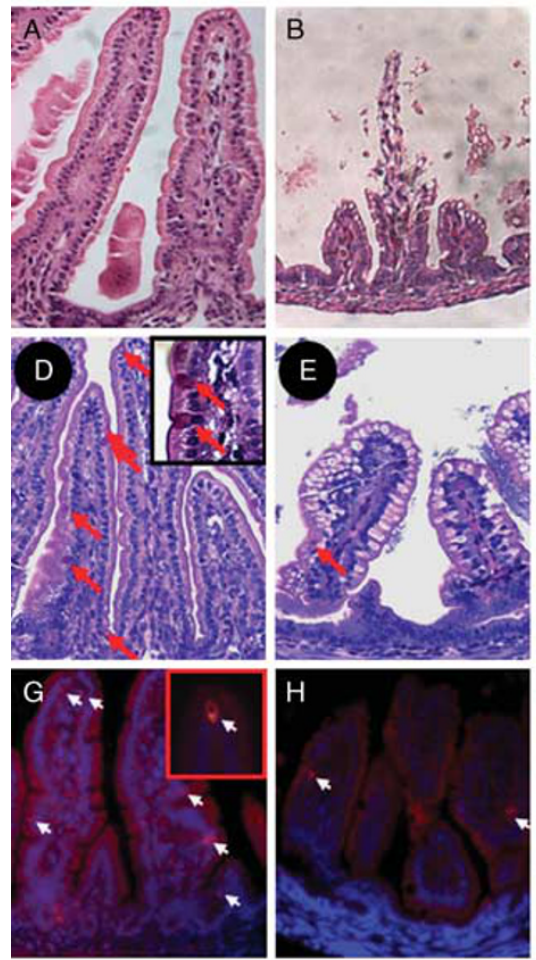

BM

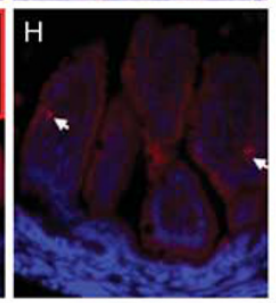

NEC
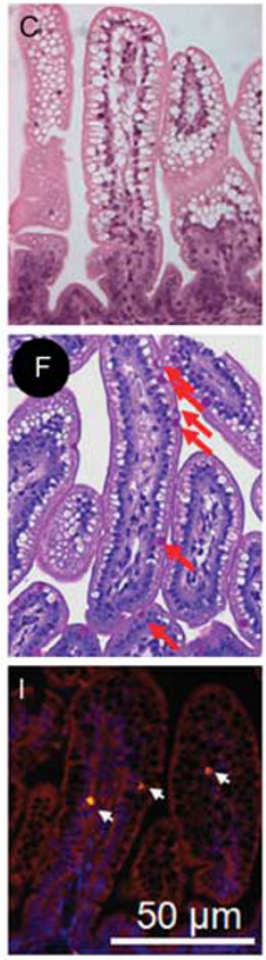

NEC+HB-EGF b
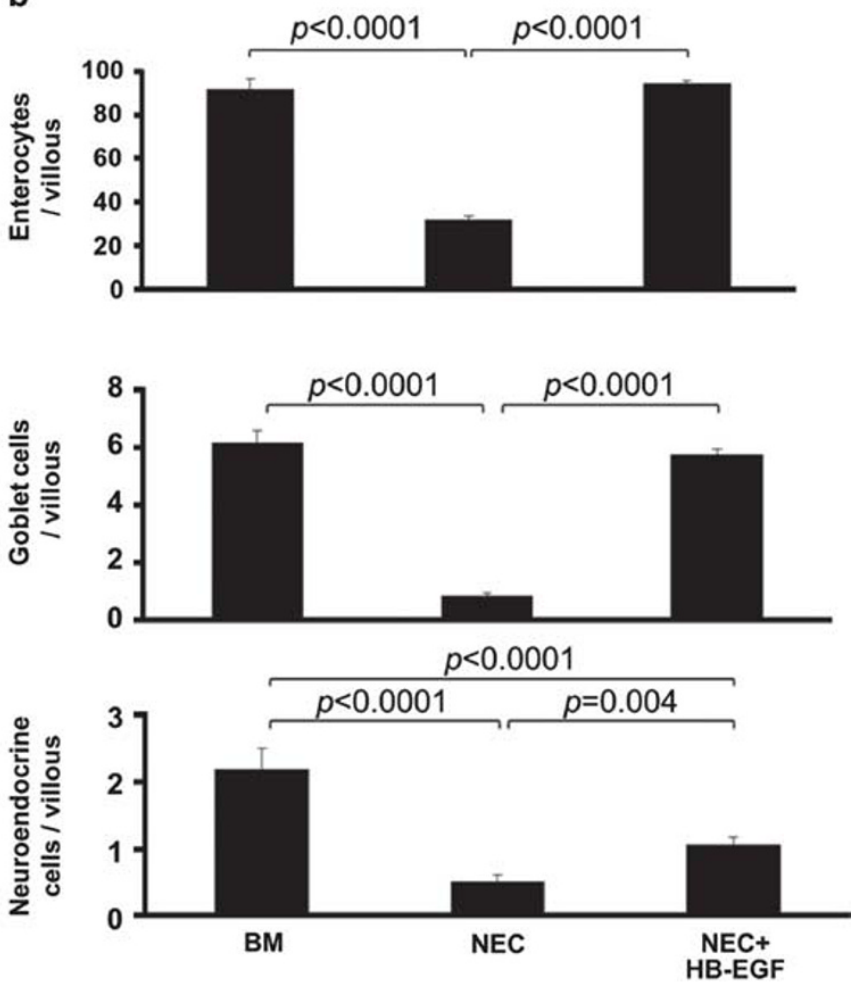

Figure 1 HB-EGF protects enterocytes, goblet cells and neuroendocrine cells from injury because of experimental NEC. (a) Representative photomicrographs of rat jejunum. (A-C) H\&E stained sections showing absorptive enterocytes; (D-F) PAS staining showing goblet cells (red arrows); and (G-I) chromogranin A immunostaining showing neuroendocrine cells (white arrows). Panels $A, D, G$ are from pups that were breast fed; panels $B$, E, $H$ are from pups subjected to experimental NEC; panels C, F, I are from pups subjected to experimental NEC but with HB-EGF added to the feeds. (b) Quantification of enterocytes, goblet cells and neuroendocrine cells. BM, pups that were breast fed by surrogate mothers; NEC, pups exposed to experimental NEC; NEC + HB-EGF, pups exposed to experimental NEC that were treated with HB-EGF added to the feeds. Values represent mean \pm s.e.m. One-way ANOVA with Tukey-Kramer pair-wise comparison test. 
a
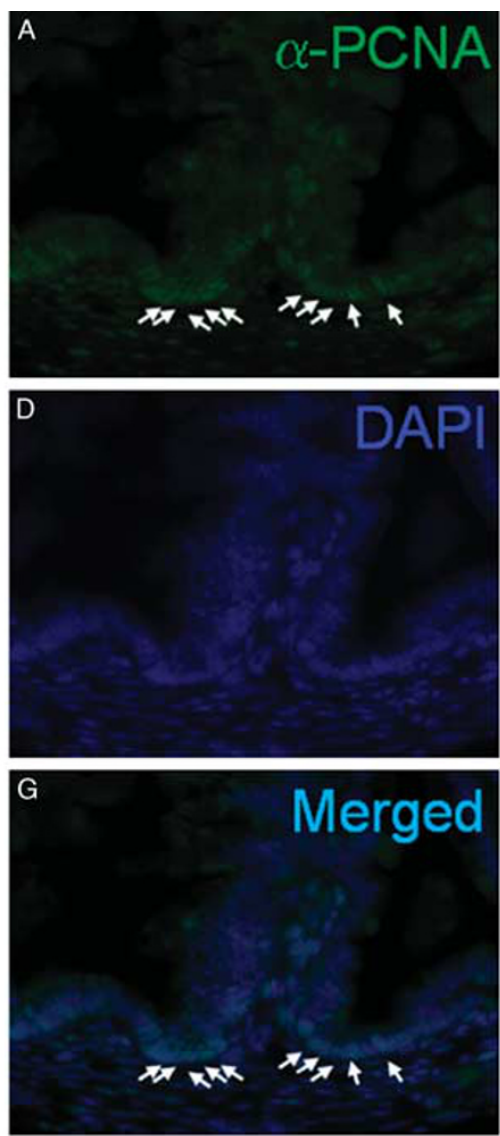

BM

b

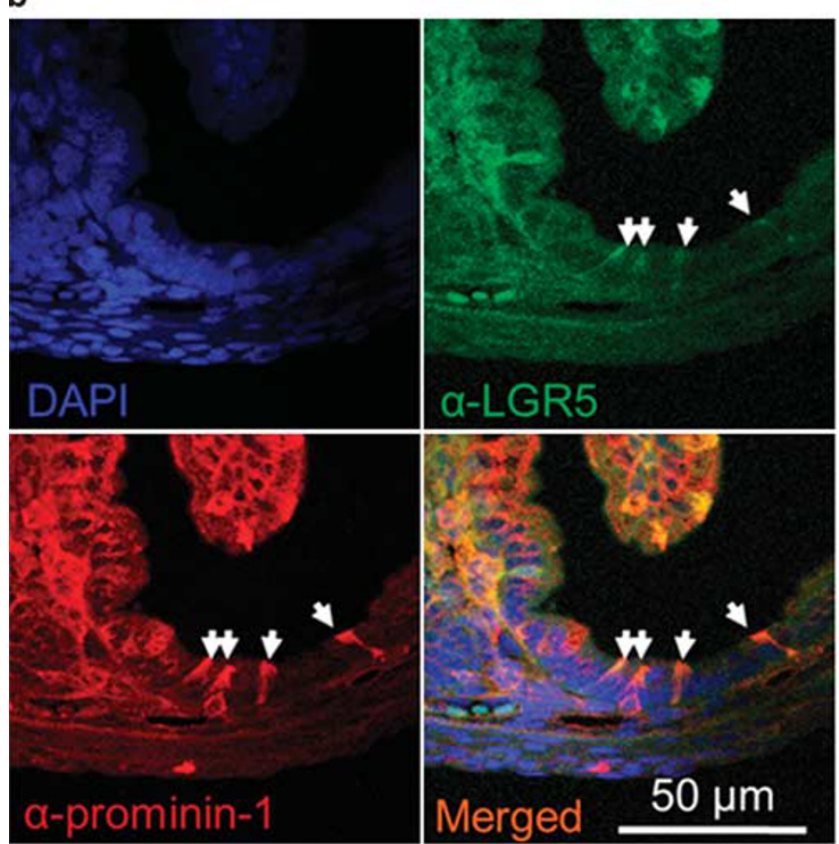

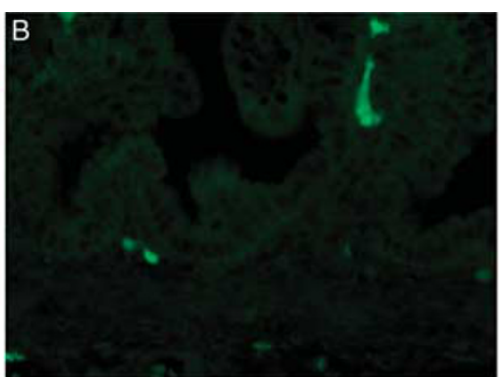
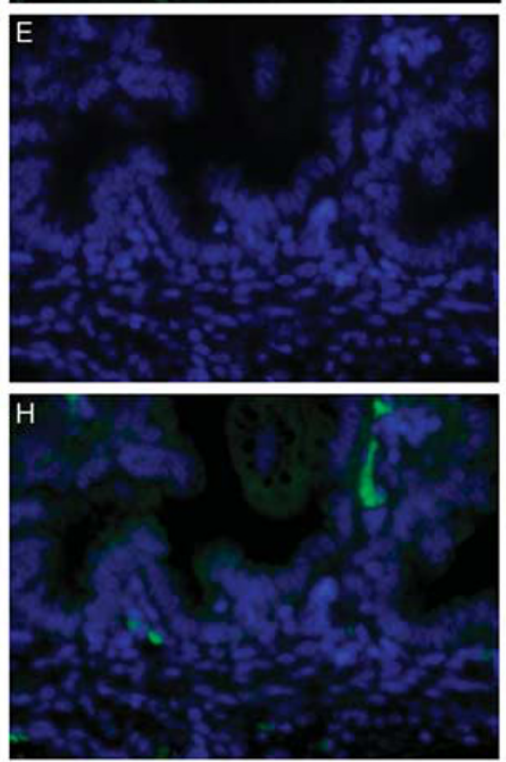

NEC
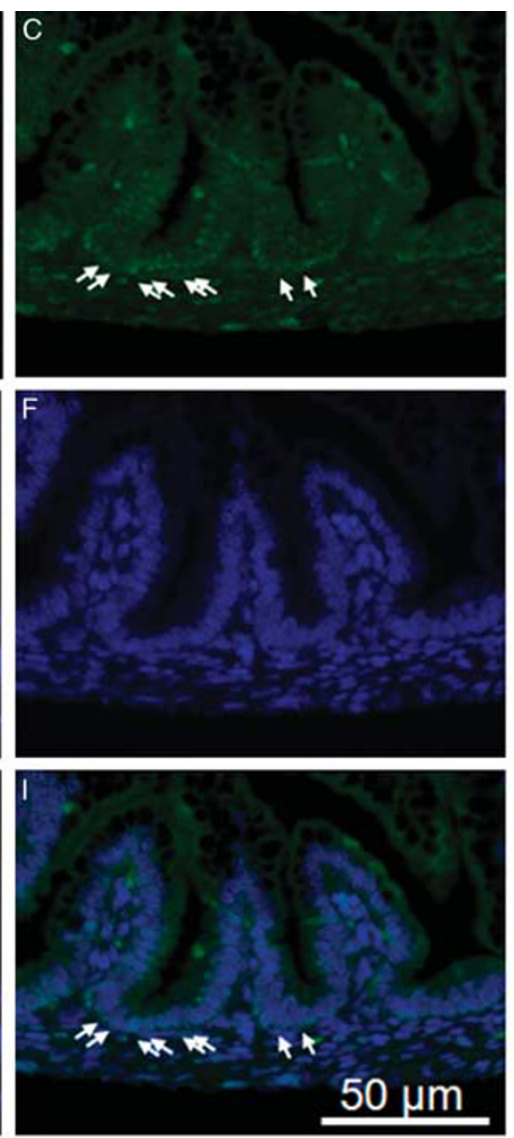

NEC+HB-EGF

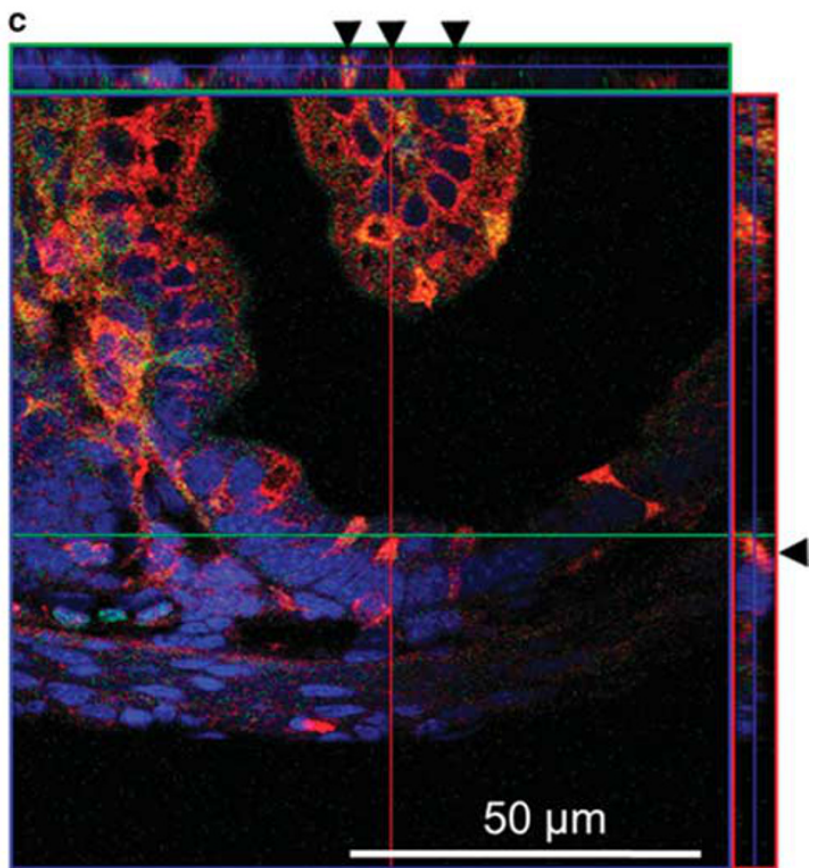

Figure 2 For caption see next page. 


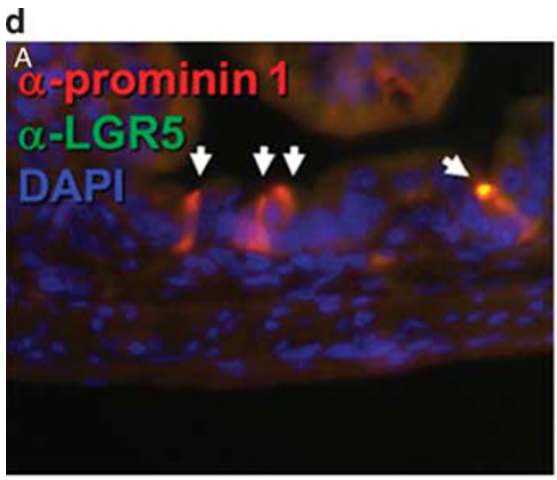

BM

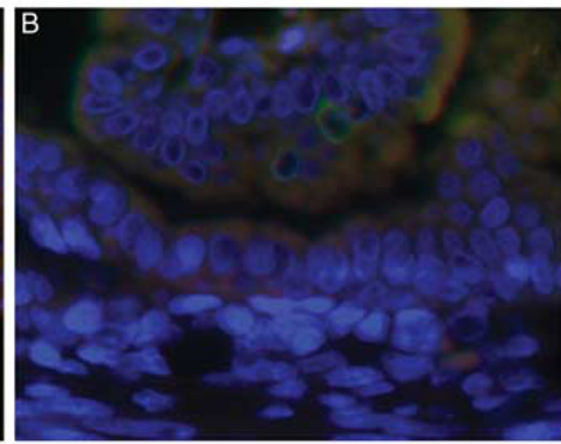

NEC

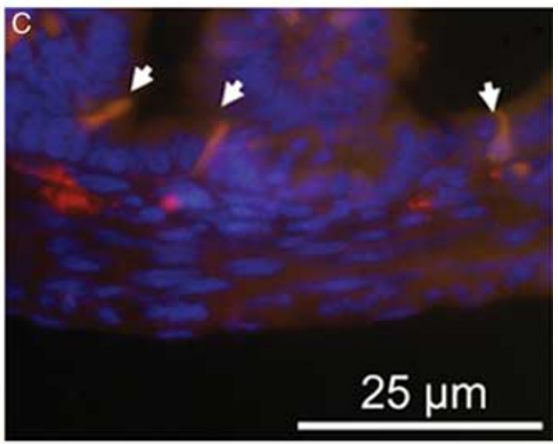

NEC+HB-EGF

E

\section{$p=0.0007$}

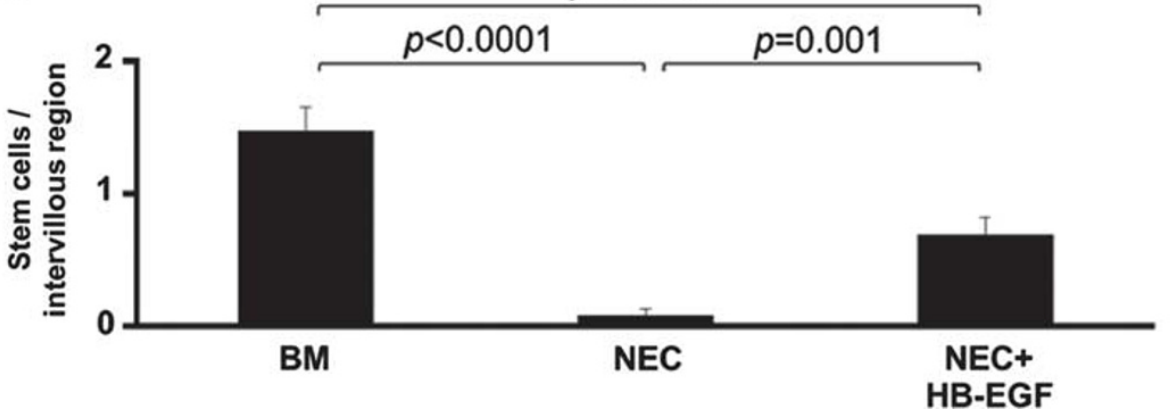

Figure 2 HB-EGF protects proliferating ISCs and TA progenitor cells from experimental NEC. (a) PCNA immunostaining of ISCs and TA progenitor cells. Shown are representative photomicrographs from: $(A, D, G)$ a breast fed rat pup; $(B, E, H)$ a rat pup exposed to experimental NEC; and $(C, F, I)$ a rat pup exposed to experimental NEC but treated with HB-EGF added to the feeds. Panels A-C are stained with an anti-PCNA antibody, panels D-F show DAPI nuclear staining, panels G-I show merged images. (b) Confocal microscopic images of anti-LGR5 and anti-prominin-1 double immunostaining of ISCs in the intervillous regions of uninjured breast-fed rat pups. (c) Confocal microscopic ortho-images of (b) display the intracellular anti-LGR5 and anti-prominin-1 double immunostaining (black arrowheads). The upper stripe shows the ortho-image along the green line and the stripe on the right shows the ortho-image along the red line. (d) HB-EGF protects ISCs from experimental NEC. Prominin-1 and LGR5 double immunostaining was used for detection of ISCs. Shown are representative photomicrographs from: (A) a breast fed rat pup; (B) a rat pup exposed to experimental NEC; and (C) a rat pup exposed to experimental NEC but with HB-EGF added to the feeds. ISCs were stained with anti-LGR5 (FITC, green) and anti-prominin-1 (Cy3, red), with DAPI nuclear staining (blue). (e) Quantification of ISCs. BM, pups breast fed by surrogate mothers; NEC, pups exposed to experimental NEC; NEC + HB-EGF, pups exposed to experimental NEC but with HB-EGF added to the feeds. Values represent mean \pm s.e.m. One-way ANOVA with Tukey-Kramer pair-wise comparison test.

conditions, we found that double immunostaining with monoclonal anti-prominin-1 and anti-LGR5 antibodies successfully identified rat pup ISCs (Figures 2b, c and Supplementary Figure 1). Prominin-1 expression in rat pup intervillous epithelial cells colocalized with LGR5 expression specific to stem cells, but not to TA progenitor cells. Confocal serial scanning confirmed that prominin-1 and LGR5 staining was both intracellular and cell membrane associated (Figure 2c and Supplementary Video 1). Some villous and mesenchymal cells stained positively, as has been described. ${ }^{5}$ We next examined the effect of HB-EGF on ISCs in our animal model of experimental NEC. The number of stem cells/intervillous region decreased significantly in pups subjected to NEC, and increased significantly in pups subjected to NEC but with HB-EGF added to the feeds (Figures $2 \mathrm{~d}$ and e). Thus, HB-EGF protects ISCs from injury in a model of experimental NEC. The decreased LGR5 expression in ISCs was also observed in human intestine resected for NEC compared with human intestine resected for small bowel atresia (Supplementary Figure 2).

\section{HB-EGF Protects Prominin-1-Positive ISCs from Hypoxic Stress In Vitro}

We next adapted an in vitro model to further investigate the cytoprotective effects of HB-EGF on ISCs. Colocalized prominin-1 and LGR5 expression in ISCs in vivo supported isolation of ISCs by $\alpha$-prominin-1 MACS, as used previously to isolate neural stem cells. ${ }^{29}$ Intervillous epithelia were separated from the villi as described in Materials and methods section (Supplementary Figure 3A), and prominin-1-positive cells were enriched by prominin-1 antibody MACS. Prominin-1 and LGR5 immunostaining confirmed $\sim 90 \%$ positively stained cells in MACS eluates compared with $<10 \%$ in flow through (Supplementary Figure 3B). Flow cytometry confirmed that $\sim 80 \%$ of the MACS purified cells expressed prominin-1 and LGR5 (Supplementary Figure 3C). In the 
absence of HB-EGF, exposure of ISCs to hypoxia led to decreased cell viability (Supplementary Figure 3D). However, addition of HB-EGF to ISCs exposed to hypoxia led to significantly increased ISC viability. Furthermore, under normoxic conditions, addition of HB-EGF also led to increased ISC viability.

\section{HB-EGF Promotes Stem Cell Viability and Growth of Crypt-Villous Organoids Ex Vivo}

We next evaluated the effects of HB-EGF on crypt-villous organoid growth ex vivo, under basal, non-injury conditions. We modified the ex vivo crypt-villous organoid culture system described by Sato et al, ${ }^{28}$ using R-spondin 1 and Noggin in the culture medium, but replacing EGF with HB-EGF. We found that crypts grew into crypt-villous organoids with a villous sphere and numerous budding crypts (Figures $3 \mathrm{a}$ and $\mathrm{b}$ ). The growth of crypt-villous organoids from the cryptal base was exponential during the 12-day culture period (Figure 3c).

Cultured organoids were designated as either viable or degraded (Figure 4a, Supplementary Videos 2A and B). The addition of R-spondin 1 alone was essential for maintenance of viable organoids, and was able to sustain organoids up to day 4 (Figures $4 \mathrm{a}$ and $\mathrm{b}$, panels $\mathrm{C}$ and $\mathrm{G}$ ). With either HBEGF alone or Noggin alone, crypts were initially viable at $12 \mathrm{~h}$ in culture, but viability dropped dramatically by days $1-2$ and was completely lost by day 4 in culture (Figures $4 \mathrm{a}$ and b, panels A, B, E and F). The addition of Noggin to R-spondin 1 did not increase the percentage of viable organoids (Figure 4a), suggesting that Noggin may not be essential for maintaining organoids, although it may be necessary for further passage of ex vivo organoid cultures. ${ }^{28}$ However, addition of HB-EGF to R-spondin 1 and Noggin significantly increased organoid viability (Figure $4 \mathrm{a}$ ), organoid size (Figures $4 \mathrm{~b}$, panels $\mathrm{D}$ and $\mathrm{H}$ and $4 \mathrm{c}$ ), and crypt fission and crypt length (Figure 4d). Together, these results indicate that HB-EGF enhances R-spondin 1-induced ISC activation and proliferation, resulting in increased organoid growth under basal, non-injury conditions. Differentiated IEC lineages were detected in cultured organoids, however, the differentiation of enterocytes, goblet cells and Paneth cells derived from ISCs (Figure 4e) and proliferative progenitor cells (Figure 4f) under these non-injury conditions did not appear to require the addition of HB-EGF.
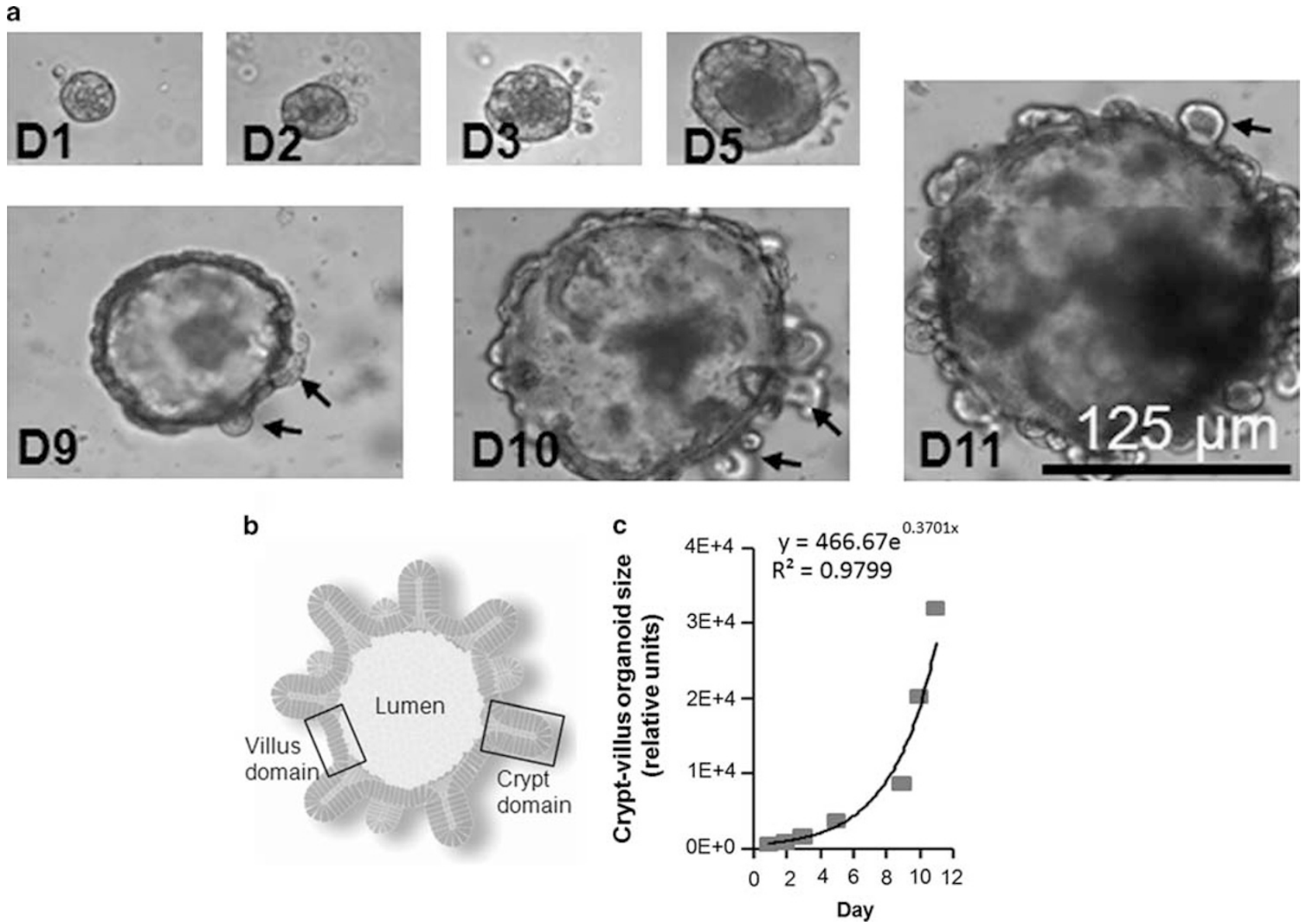

Figure 3 Ex vivo crypt-villous organoid cultures. (a) A representative mouse crypt cultured ex vivo in gelmatrix that grew into a crypt-villous organoid with budding crypts (black arrows) extending from the surface at days 1-11. (b) Organoid illustration showing lumen, villus domain, crypt domain and ISCs in green (from Sato et $a^{28}$ ). (c) Exponential growth of crypt-villous organoids cultured with R-spondin 1, Noggin and HB-EGF (50 ng/ml). 


\section{HB-EGF Protects Ex Vivo Crypt-Villous Organoids from Hypoxic Injury Via EGFR Activation and the MEK1/2 Signaling Pathway}

To investigate the effects of HB-EGF on ISC survival and proliferation on exposure to injury, the sizes and the percentage of viable organoids were quantified in ex vivo crypt- villous organoid cultures exposed to normoxia or hypoxia for $60 \mathrm{~min}$. In the absence of HB-EGF, organoid size remained static under normoxic or hypoxic conditions at all-time points tested (Figure 5a). However, crypt-villous organoid growth in the presence of HB-EGF was significantly increased at 3 and 5 days after exposure to either hypoxia or normoxia.
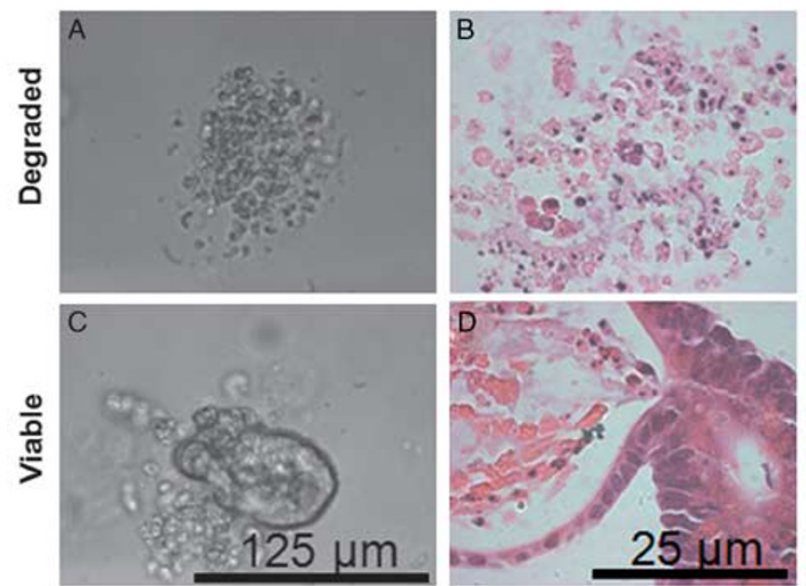

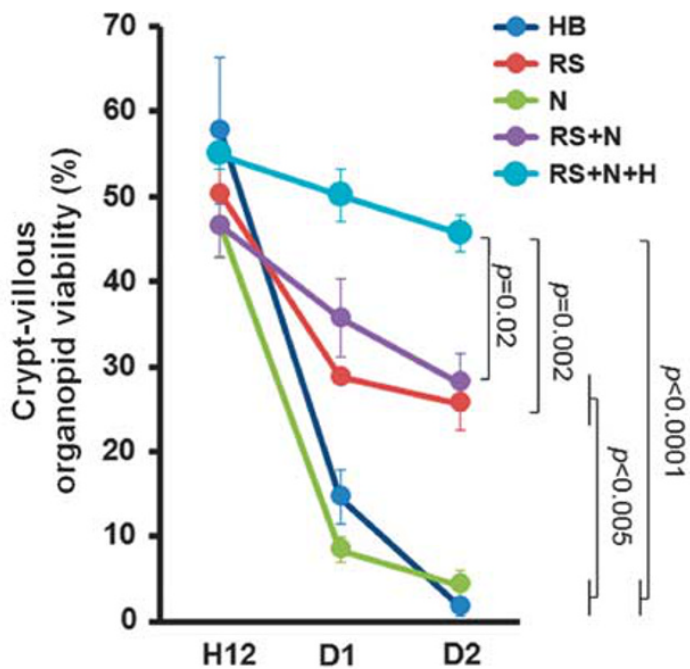

b
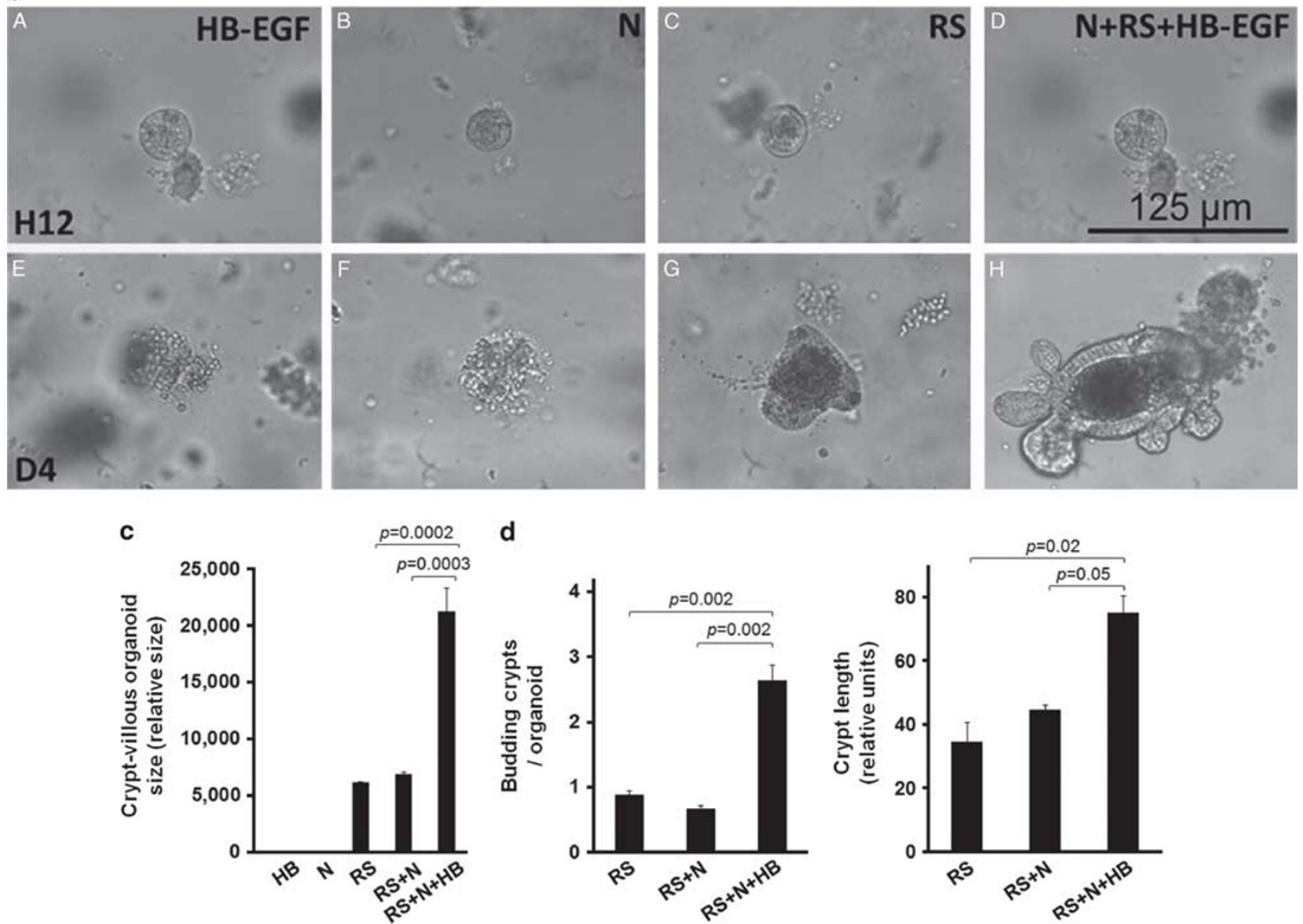

Figure 4 For caption see next page. 
e

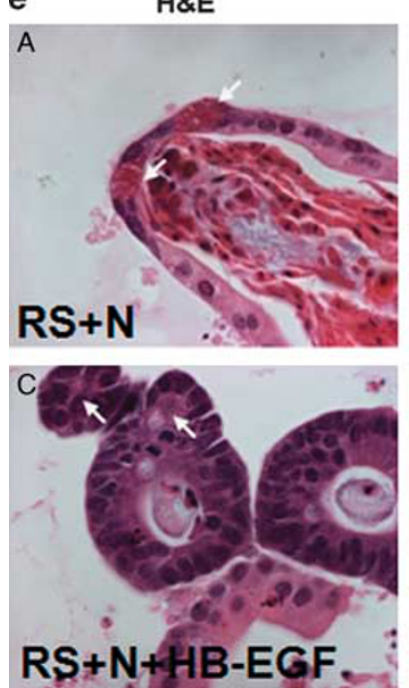

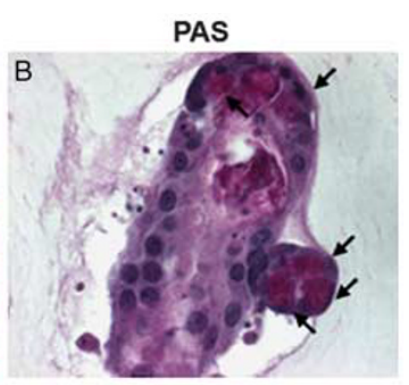

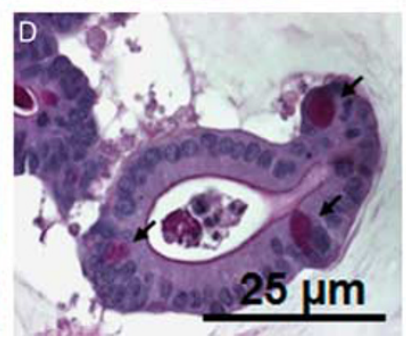

f
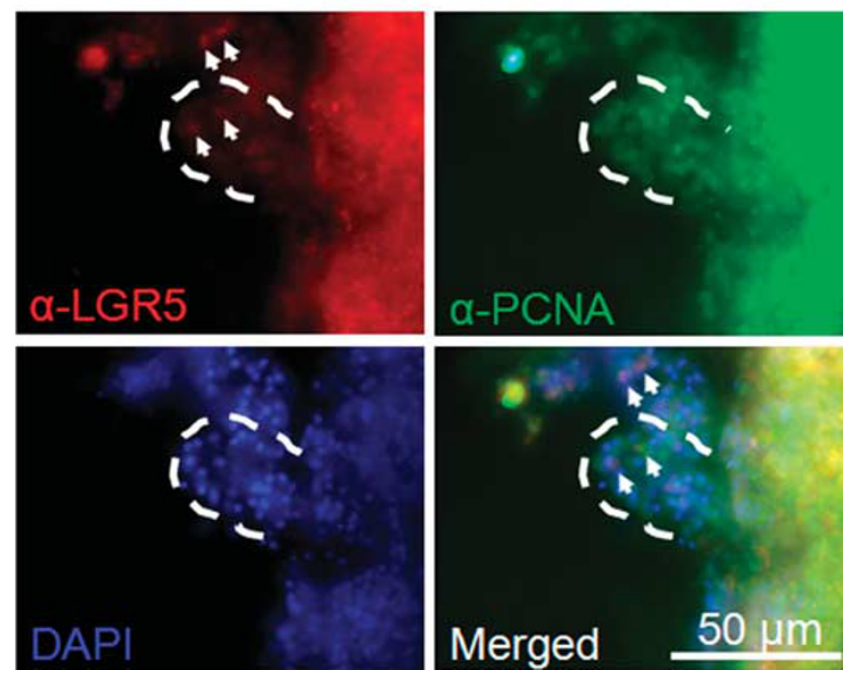

Figure 4 HB-EGF protects crypt-villous organoids from hypoxia. (a) Representative photomicrographs of viable and degraded organoids as visualized by phase contrast microscopy (panels $A, C$ ) and H\&E staining (panels $B, D)$. Percentage of viable organoids with different culture additives at $12 \mathrm{~h}$ (H12), day 1 (D1) and day 2 (D2). (b) Representative crypt-villous organoids cultured for 12 h or 4 days in medium containing: (A, E) HB-EGF; (B, F) Noggin; (C, G) R-Spondin 1; (D, H) Noggin + R-spondin $1+$ HB-EGF. Panels A-D: 12 h; panels E-H: day 4. (c) Size of crypt-villous organoids cultured with different additives on day 4. (d) Budding crypts/crypt-villous organoid cultured with different additives on day 4 and budding crypt length cultured with different additives on day 4. (e) Crypt-villous organoids (day 4) cultured with R-spondin and Noggin, in the absence (panels A, B) or presence (panels C, D) of HB-EGF (50 ng/ml). Panels A, C: H\&E staining; panels B, D: PAS staining; white arrows, Paneth cells; black arrows, goblet cells. (f) Crypt-villous organoids (day 4) cultured with R-spondin and Noggin and HB-EGF (50 ng/ml), and immunostained with anti-LGR5 (Cy3, red) and anti-PCNA (Cy2, green), to identify ISCs and TA progenitor cells, respectively. The white dashed lines indicate budding crypts and the white arrows indicate ISCs at the base of the crypts. In a-e: RS, R-spondin 1; N, Noggin; HB, HB-EGF. In b, c: values represent mean \pm s.e.m. (b; right panel), two-way ANOVA; (b; left panel) and (c), one-way ANOVA with Tukey-Kramer pair-wise comparison test.

HB-EGF significantly increased the percentage of viable organoids at days 1, 2 and 3 under normoxic conditions, and at day 3 on exposure to hypoxia (Figure 5b). This indicates that HB-EGF protects ISCs from hypoxic injury and promotes ISC proliferation even under hypoxic conditions.

Signal pathway inhibitor studies suggest that HB-EGF promotes crypt-villous organoid proliferation via activation of EGFR/MEK1/2 and PI3K/Akt signaling pathways (Figures 5c-e and 6; Supplementary Figure 4). In the absence of inhibitors, crypts grew into crypt-villous organoids in the presence of HB-EGF beginning at day 1 (Figure $5 \mathrm{c}$, panels $\mathrm{A}$ and F; Supplementary Video 2A). In the presence of specific inhibitors to EGFR, PI3K or MEK1/2 signaling, organoid size (Figure 5d) and viability (Figure 5c, panels B-E and G-J and Figure 5e) were significantly decreased. Organoids cultured in the presence of HB-EGF and the MEK1/2 inhibitor were composed of a cellular sphere with none to few shortened protruding crypts (Figure $5 c$, panels D and I, Figures $5 \mathrm{~d}$ and 6) similar to organoids grown without HB-EGF (Figures $4 \mathrm{~b}$, panel G). Organoids cultured in the presence of HB-EGF and the EGFR inhibitor (Figure 5c, panels B and G and Figure 6) or the $\mathrm{PI} 3 \mathrm{~K}$ inhibitor (Figure $5 c$, panels $\mathrm{C}$ and $\mathrm{H}$ and Figure 6) suffered more severe consequences. Under these conditions, organoids stopped growing by day 1 , and were completely degraded into debris by days $2-5$ (Figures $5 \mathrm{~d}$, e and 6 ). These findings were similar under either normoxic or hypoxic conditions.

\section{DISCUSSION}

The lining of the intestines is composed of millions of villi and crypts, which form a barrier against bacterial invasion. The intestinal epithelium is the most rapidly proliferating tissue in adult mammals. ISCs are responsible for selfrenewal of the epithelium, and also represent a reserve pool of cells that can be activated after injury. The estimated number of stem cells is $4-6$ per crypt. ${ }^{3}$ Stem cells have been proven to be crucial for the recovery and regeneration of several tissues including the intestinal epithelium. ${ }^{36,37}$

Our previous studies have shown that HB-EGF protects the intestines in several animal models of intestinal injury including ischemia/reperfusion injury, ${ }^{38}$ hemorrhagic shock and resuscitation, ${ }^{18}$ and NEC. ${ }^{10,11,39}$ Our previous studies showed that administration of HB-EGF promotes enterocyte migration, ${ }^{15}$ prevents IEC apoptosis, ${ }^{15}$ preserves gut barrier function $^{18}$ and prevents bacterial translocation. ${ }^{17}$ The current studies show that HB-EGF can protect ISCs, TA progenitor cells and differentiated IEC cell lineages from injury in a rat pup model of NEC. The ability of HB-EGF to protect pluripotent ISCs is critical because these cells undergo selfrenewal and proliferation to replenish cells that are lost to shedding during normal homeostasis or to injury during stress. $^{4,40}$

In the past, ISCs were identified at position +4 from the crypt bottom, directly above the Paneth cells. It is now 

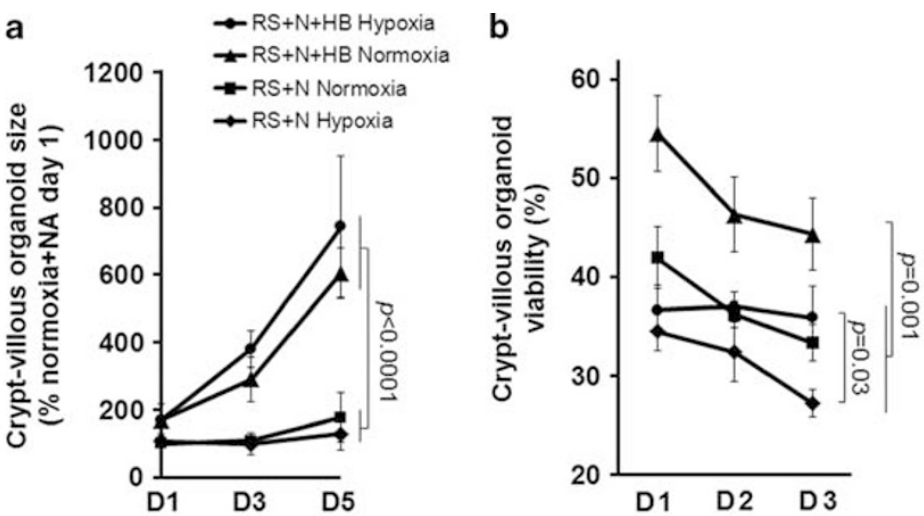

C

Normoxia

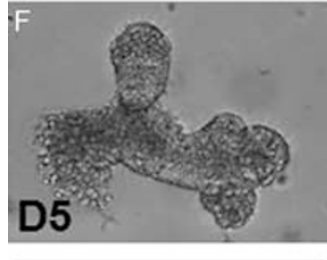

No inh
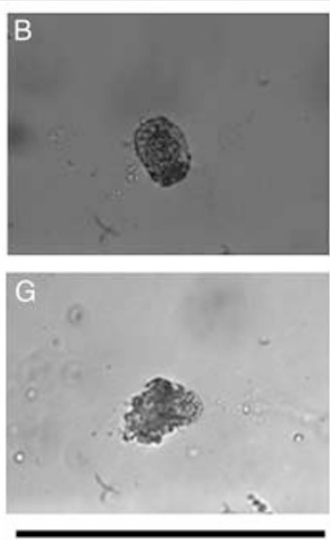

EGFR inh
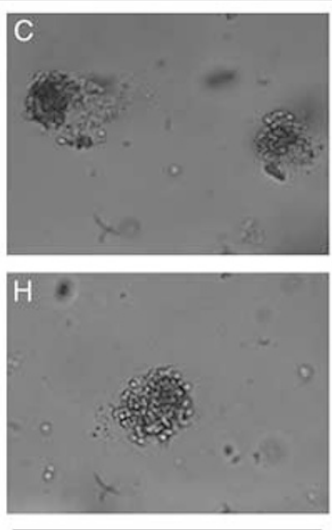

PI3K inh
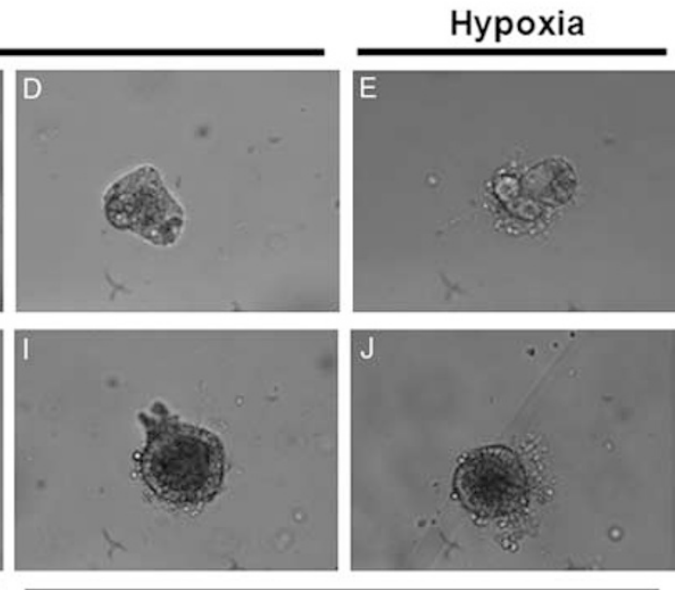

Mek $1 / 2$ inh d

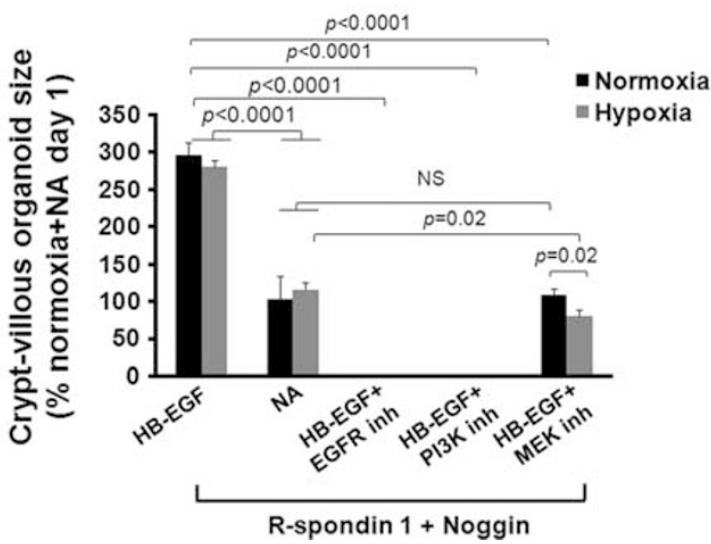

e

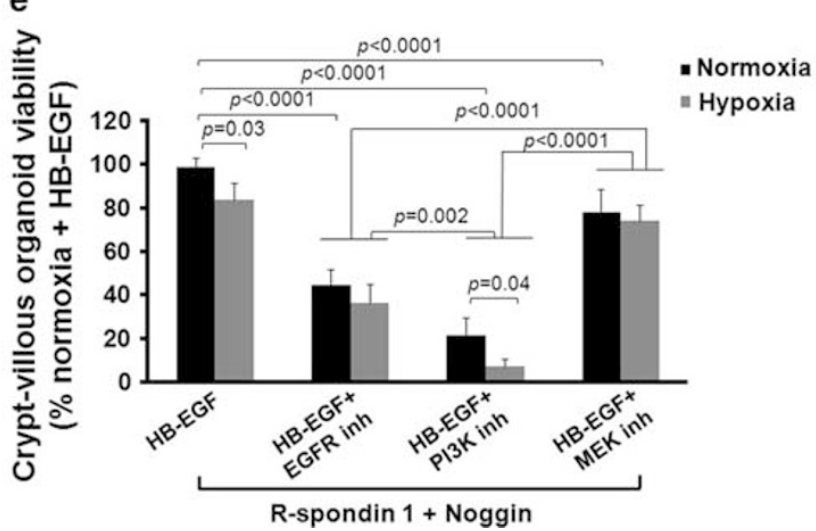

Figure 5 HB-EGF promotes crypt proliferation under hypoxic stress via EGFR activation and PI3K/Akt and MEK1/2 pathways. (a) Organoid size after exposure to hypoxia for $60 \mathrm{~min}$ followed by culture for 1-5 days in the presence of R-spondin 1 and Noggin, with or without HB-EGF (50 ng/ml).

(b) Percentage of viable organoids after exposure to hypoxia for $60 \mathrm{~min}$ followed by culture for 1-3 days in the presence of R-spondin 1 and Noggin, with or without HB-EGF $(50 \mathrm{ng} / \mathrm{ml})$. (c) Representative photomicrographs of crypt-villous organoids cultured in the presence of R-spondin 1, Noggin and HB-EGF (50 ng/ml), plus the addition of: (A, F) no inhibitors; (B, G) AG 1487 (EGFR inhibitor); (C, H) LY294002 (PI3K inhibitor); (D, E, l, J) PD98059 (MEK1/2 inhibitor). Panels A-E represent day 1 of culture; panels $F-J$ represent day 5 of culture. The organoids in panels $E$ and $J$ were exposed to hypoxia (100\% nitrogen for $60 \mathrm{~min}$ ); the organoids in all other panels were exposed to normoxia. (d) Organoid size on day 5 of culture, in the presence of R-spondin 1 and Noggin and HB-EGF, with or without inhibitors, on exposure to hypoxia or normoxia. (e) Quantification of viable organoids on day 1 of culture, in the presence of R-spondin 1 and Noggin and HB-EGF, with or without inhibitors, on exposure to hypoxia or normoxia. NA, medium containing R-spondin 1 and Noggin with no addition of HB-EGF; N, normoxia; H, hypoxia; D1, day 1 of culture; D2, day 2; D3, day 3; D5, day 5. Values represent mean \pm s.e.m. Two-way ANOVA with Tukey-Kramer pair-wise comparison test. 


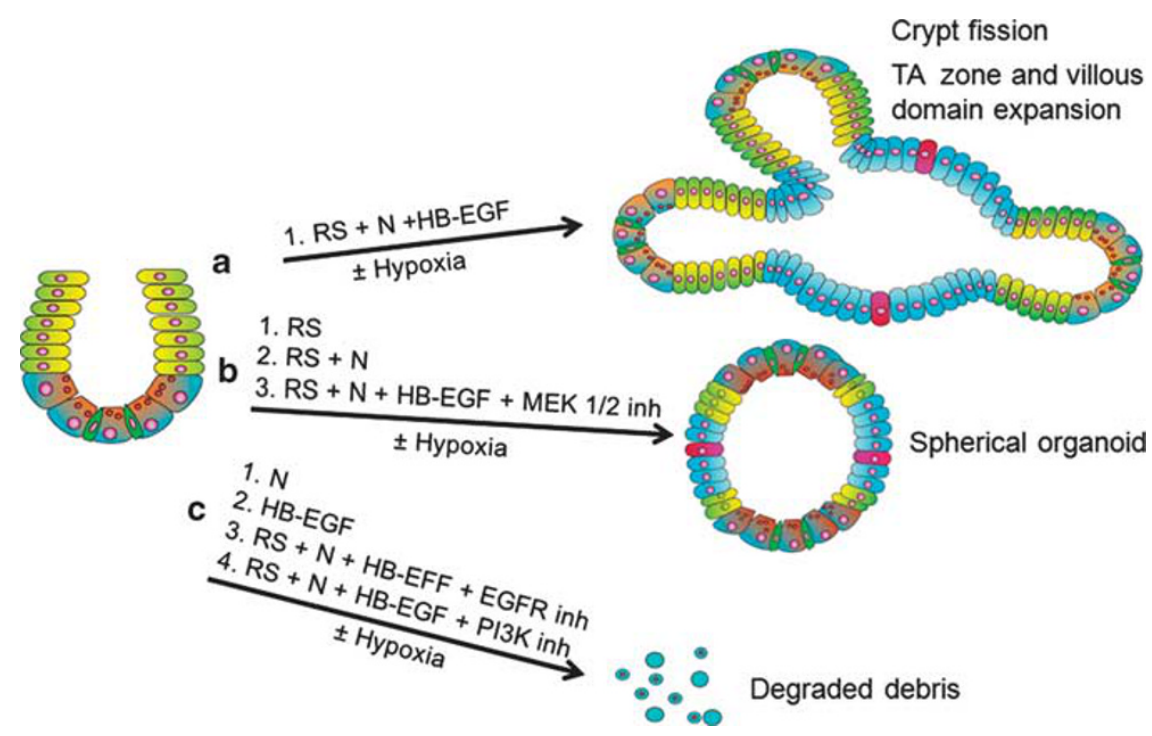

Figure 6 Schematic summary of ex vivo organoid culture experiments. (a) In the presence of R-spondin 1 and Noggin, addition of HB-EGF leads to increased crypt-villous organoid growth, with promotion of crypt fission and TA zone/villous domain expansion. (b) In the presence of R-spondin 1 alone, R-spondin 1 plus Noggin, or R-spondin 1 plus Noggin plus HB-EGF in the presence of MEK 1/2 inhibition, crypt-villous organoid growth is limited to small spherical organoids. (c) In the presence of Noggin alone, HB-EGF alone, or R-spondin 1 plus Noggin plus HB-EGF in the presence of either EGFR or PI3K inhibition, crypt-villous organoid growth is completely abolished. Thus, EGFR and PI3K activation are crucial, and MEK $1 / 2$ activation is important, in HB-EGF-mediated crypt-villous organoid growth. ISCs, green; progenitor cells in TA zone, yellow; Paneth cells, brown; enterocytes, blue; goblet cells, red. N, Noggin; RS, R-spondin 1.

thought that there may be two populations of ISCs, a slowly cycling quiescent reserve population above the Paneth cells (upper stem cell zone, USZ) (the +4 cells), and a more rapidly cycling (every $24 \mathrm{~h}$ ) active pool of crypt base columnar (CBC) cells located between the Paneth cells (lower stem cell zone, LSZ). The more active ISCs may maintain homeostatic regenerative capacity of the intestine with the more quiescent ISCs held in reserve. ${ }^{7}$ Several signaling pathways including the $\mathrm{Wnt} / \beta$-catenin, BMP, RTK/PI3K and Notch cascades are critical to ISC self-renewal and proliferation. Among them, $\mathrm{Wnt} / \beta$-catenin is the signature signaling pathway, and its downstream regulated genes represent potential ISC markers. The $\mathrm{Wnt} / \beta$-catenin target gene LGR5 has been recently identified as a marker for CBC ISCs. ${ }^{28}$ Prominin-1 is also expressed in ISC. ${ }^{6,27}$

Evidence that $\mathrm{Wnt} / \beta$-catenin signaling is critical to ISC proliferation includes the observations that ISCs accumulate nuclear $\beta$-catenin, a hallmark of active Wnt signaling, and that abrogation of Wnt signaling leads to loss of ISC proliferation. ${ }^{7,37}$ The canonical Wnt pathway is activated when Wnt ligands such as $\mathrm{R}$ spondin 1 bind to the cell surface Frizzled/LRP co-receptor complex (Supplementary Figure 4). Axin is recruited to the cell membrane resulting in inactivation of the APC destruction complex and stabilization of $\beta$-catenin when PI3K/AKT facilitates $\beta$-catenin C-terminal S552 phosphorylation and GSK3 $\beta$ phosphorylation. Whereas active Wnt signaling promotes proliferation of ISCs, signaling of the TGF- $\beta$ family member BMP maintains ISC quiescence. BMP activation leads to SMAD and PTEN signaling, with suppression of ISC proliferation.
Unphosphorylated PTEN is active and inhibits Akt activation; phosphorylated PTEN is inactive leading to Akt activation. Inhibition of BMP signaling by overexpression of its inhibitor Noggin, or inactivation of its receptor BMPR1A, causes ectopic crypt formation, suggesting a role for BMP in restricting crypt numbers. Activated $\beta$-catenin translocates to the nucleus, binds to the Tcf transcription complex and promotes the expression of pro-cell-cycling c-Myc, cyclin D and growth factors that lead to ISC self-renewal and proliferation, and ISC markers including LGR5. ${ }^{5}$

In the current studies, loss of the stem cell markers LGR5 and prominin- 1 in the intervillous regions of pups subjected to NEC suggests aberrant changes in Wnt signaling, and indicates either an abnormality in the ISCs or physical disappearance of the cells, or both. Treatment with HB-EGF restored LGR5 and prominin-1 expression in ISCs in rat pups exposed to NEC. In addition, HB-EGF was found to be protective for isolated ISCs subjected to hypoxic stress in vitro. Furthermore, in ex vivo crypt-villous organoid cultures, addition of HB-EGF led to ISC proliferation, expansion of the cryptal TA compartment resulting in elongated crypts, and growth of crypt-villous organoids, even in the face of hypoxia. Collectively, these findings strongly support the notion that HB-EGF protects the intestine from injury, at least in part, through promotion of ISC/progenitor cell viability and proliferation.

HB-EGF appears to exert its protection of ISCs and TA progenitor cells through the PI3K and EGFR/MEK1/2/ ERK1/2 signaling pathways under conditions of normoxia or hypoxia. R-spondin 1 is a Wnt agonist that acts as a secreted 
activator of $\mathrm{Wnt} / \beta$-catenin signaling and promotes hyperproliferation of crypts. ${ }^{41}$ Our ex vivo crypt culture experiments demonstrate that HB-EGF or Noggin alone cannot sustain ISC viability, and that R-Spondin 1 -initated $\mathrm{Wnt} / \beta$ catenin signaling is required for the maintenance of longterm organoid cultures. As HB-EGF and Noggin are each able to activate PI3K signaling, this indicates that activation of the PI3K cascade alone is not sufficient to sustain ISC survival and proliferation. However, our results with PI3K inhibitors indicate that $\mathrm{PI} 3 \mathrm{~K}$ signaling has an important complementary role to the $\mathrm{Wnt} / \beta$-catenin cascade in promoting ISC viability under normoxia or hypoxia. In our ex vivo crypt cultures grown in the presence of R-spondin 1 alone, PI3K signaling is likely provided by a $\mathrm{Wnt} / \beta$-catenin-dependent autocrine growth factor loop (Supplementary Figure 4). Another signaling cascade that HB-EGF utilizes to exert its effects on ISCs is the EGFR/MEK1/2/ERK1/2 pathway. When added to R-spondin and Noggin, HB-EGF enhances the viability of isolated ISC in vitro and the growth of organoids cultured ex vivo. These effects of HB-EGF are significantly compromised in the presence of MEK1/2 inhibition, demonstrating that EGFR/MEK1/2 is a main execution pathway for HB-EGF-mediated ISC proliferation. MEK 1/2/ERK1/2 are activated and regulated by RTK/Ras/MEKs ${ }^{42}$ to promote cell proliferation via activation of the cAMP response element-binding protein, E-twenty-six and c-fos transcription factors, resulting in cyclin D1 induction or inhibition of cell cycle inhibitors such as p27kip1, Max dimerization protein 1 and Myt1. ${ }^{43}$ MEK1/2/ERK1/2 also enhance survival through $90 \mathrm{kDa}$ ribosomal S6 kinase, which inactivates the proapoptotic protein BAD. Exactly how the EGFR transmits HB-EGF signaling to MEK1/2 and what the target genes are remain to be elucidated, but Ras is a potential mediator. It has been demonstrated that signaling through the EGFR/RAS/MEK1/2 pathway is necessary and limiting for adult midgut progenitor cell proliferation. ${ }^{44}$ Additionally, MEK1/2 activation is associated with proliferation and survival in mouse embryonic and adult ISCs incubated with EGF in vitro. ${ }^{45}$

In summary, the current studies show that HB-EGF protects ISCs from hypoxic injury in vitro, and from injury because of experimental NEC in vivo. Future studies will examine the ability of exogenously administered HB-EGF and ISCs, delivered alone and in combination, to protect the intestines from NEC. Our findings lend further support for the administration of HB-EGF to premature babies at greatest risk of developing NEC, in an effort to prevent or treat this often devastating disease.

Supplementary Information accompanies the paper on the Laboratory Investigation website (http://www.laboratoryinvestigation.org)

\section{ACKNOWLEDGEMENT}

We thank for Dr Christopher Pierson for assistance with attempts at Paneth cell identification. This work was supported by NIH R01 DK74611 and NIH R01 GM61193 (GEB).

\section{DISCLOSURE/CONFLICT OF INTEREST}

The authors declare no conflict of interest.

1. Lee JS, Polin RA. Treatment and prevention of necrotizing enterocolitis. Semin Neonatol 2003;8:449-459.

2. Ballance WA, Dahms BB, Shenker N, et al. Pathology of neonatal necrotizing enterocolitis: a ten-year experience. J Pediatr 1990;117:S6-13.

3. Barker N, Clevers H. Tracking down the stem cells of the intestine: strategies to identify adult stem cells. Gastroenterology 2007;133: 1755-1760.

4. Potten CS, Gandara R, Mahida YR, et al. The stem cells of small intestinal crypts: where are they? Cell Prolif 2009;42:731-750.

5. Barker N, van Es JH, Kuipers J, et al. Identification of stem cells in small intestine and colon by marker gene Lgr5. Nature 2007;449:1003-1007.

6. Snippert $\mathrm{HJ}$, van Es JH, van den Born $\mathrm{M}$, et al. Prominin-1/CD133 marks stem cells and early progenitors in mouse small intestine. Gastroenterology 2009;136:2187-2194.

7. Scoville DH, Sato T, He XC, et al. Current view: intestinal stem cells and signaling. Gastroenterology 2008;134:849-864.

8. Markel TA, Crisostomo PR, Lahm T, et al. Stem cells as a potential future treatment of pediatric intestinal disorders. J Pediatr Surg 2008;43: 1953-1963.

9. Gupta A, Dixit A, Sales KM, et al. Tissue engineering of small intestinecurrent status. Biomacromolecules 2006;7:2701-2709.

10. Feng J, El-Assal ON, Besner GE. Heparin-binding EGF-like growth factor (HB-EGF) and necrotizing enterocolitis. Semin Pediatr Surg 2005;14:167-174.

11. Radulescu A, Zorko NA, Yu X, et al. Preclinical neonatal rat studies of heparin-binding EGF-like growth factor in protection of the intestines from necrotizing enterocolitis. Pediatr Res 2009;65:437-442.

12. Besner GE, Klagsbrun M. Macrophages secrete a heparin-binding inhibitor of endothelial cell growth. Microvasc Res 1991;42:187-197.

13. Higashiyama S, Abraham JA, Miller J, et al. A heparin-binding growth factor secreted by macrophage-like cells that is related to EGF. Science 1991;251:936-939.

14. Nanba D, Mammoto A, Hashimoto $K$, et al. Proteolytic release of the carboxy-terminal fragment of proHB-EGF causes nuclear export of PLZF. J Cell Biol 2003;163:489-502.

15. Feng J, Besner GE. Heparin-binding epidermal growth factor-like growth factor promotes enterocyte migration and proliferation in neonatal rats with necrotizing enterocolitis. J Pediatr Surg 2007;42: 214-220.

16. Feng J, El-Assal ON, Besner GE. Heparin-binding epidermal growth factor-like growth factor reduces intestinal apoptosis in neonatal rats with necrotizing enterocolitis. J Pediatr Surg 2006;41:742-747.

17. Xia G, Martin AE, Michalsky MP, et al. Heparin-binding EGF-like growth factor preserves crypt cell proliferation and decreases bacterial translocation after intestinal ischemia/reperfusion injury. J Pediatr Surg 2002;37:1081-1087.

18. El-Assal ON, Radulescu A, Besner GE. Heparin-binding EGF-like growth factor preserves mesenteric microcirculatory blood flow and protects against intestinal injury in rats subjected to hemorrhagic shock and resuscitation. Surgery 2007;142:234-242.

19. Rocourt DV, Mehta VB, Besner GE. Heparin-binding EGF-like growth factor decreases inflammatory cytokine expression after intestinal ischemia/reperfusion injury. J Surg Res 2007;139:269-273.

20. Xia G, Martin AE, Besner GE. Heparin-binding EGF-like growth factor downregulates expression of adhesion molecules and infiltration of inflammatory cells after intestinal ischemia/reperfusion injury. J Pediatr Surg 2003;38:434-439.

21. Barlow B, Santulli TV. Importance of multiple episodes of hypoxia or cold stress on the development of enterocolitis in an animal model. Surgery 1975;77:687-690.

22. Yu X, Radulescu A, Zorko N, et al. Heparin-binding EGF-like growth factor increases intestinal microvascular blood flow in necrotizing enterocolitis. Gastroenterology 2009;137:221-230.

23. Cetin S, Ford HR, Sysko LR, et al. Endotoxin inhibits intestinal epithelial restitution through activation of Rho-GTPase and increased focal adhesions. J Biol Chem 2004;279:24592-24600.

24. Caplan MS, Hedlund $E$, Adler $L$, et al. Role of asphyxia and feeding in a neonatal rat model of necrotizing enterocolitis. Pediatr Pathol 1994;14: 1017-1028. 
25. Trahair JF, Perry RA, Silver $M$, et al. Autoradiographic localization of $(3 \mathrm{H})$-thymidine incorporation in the small intestinal epithelium of fetal sheep. J Pediatr Gastroenterol Nutr 1986;5:648-654.

26. Lee $A$, Kessler JD, Read TA, et al. Isolation of neural stem cells from the postnatal cerebellum. Nat Neurosci 2005;8:723-729.

27. Zhu L, Gibson P, Currle DS, et al. Prominin 1 marks intestinal stem cells that are susceptible to neoplastic transformation. Nature 2009;457: 603-607.

28. Sato T, Vries RG, Snippert HJ, et al. Single Lgr5 stem cells build cryptvillus structures in vitro without a mesenchymal niche. Nature 2009; 459:262-265.

29. Yu S, Zhang JZ, Zhao CL, et al. Isolation and characterization of the CD133+ precursors from the ventricular zone of human fetal brain by magnetic affinity cell sorting. Biotechnol Lett 2004;26:1131-1136.

30. Dekaney CM, Rodriguez JM, Graul MC, et al. Isolation and characterization of a putative intestinal stem cell fraction from mouse jejunum. Gastroenterology 2005;129:1567-1580.

31. Bjerknes $\mathrm{M}$, Cheng $\mathrm{H}$. Methods for the isolation of intact epithelium from the mouse intestine. Anat Rec 1981;199:565-574.

32. Levitzki A. Protein tyrosine kinase inhibitors as novel therapeutic agents. Pharmacol Ther 1999;82:231-239.

33. Vlahos CJ, Matter WF, Hui KY, et al. A specific inhibitor of phosphatidylinositol 3-kinase, 2-(4-morpholinyl)-8-phenyl-4H-1benzopyran-4-one (LY294002). J Biol Chem 1994;269:5241-5248.

34. Pang L, Sawada T, Decker SJ, et al. Inhibition of MAP kinase kinase blocks the differentiation of PC-12 cells induced by nerve growth factor. J Biol Chem 1995;270:13585-13588.
35. Chen $\mathrm{CL}$, Mehta VB, Zhang $\mathrm{HY}$, et al. Intestinal phenotype in mice overexpressing a heparin-binding EGF-like growth factor transgene in enterocytes. Growth Factors 2010;28:82-97.

36. Vaananen HK. Mesenchymal stem cells. Ann Med 2005;37:469-479.

37. Barker $\mathrm{N}$, van de Wetering $\mathrm{M}$, Clevers $\mathrm{H}$. The intestinal stem cell. Genes Dev 2008;22:1856-1864.

38. El-Assal ON, Besner GE. HB-EGF enhances restitution after intestinal ischemia/reperfusion via PI3K/Akt and MEK/ERK1/2 activation. Gastroenterology 2005;129:609-625.

39. Feng J, El-Assal ON, Besner GE. Heparin-binding epidermal growth factor-like growth factor decreases the incidence of necrotizing enterocolitis in neonatal rats. J Pediatr Surg 2006:41:144-149.

40. Haegebarth $A$, Clevers $H$. Wnt signaling, lgr5, and stem cells in the intestine and skin. Am J Pathol 2009;174:715-721.

41. Kim KA, Kakitani M, Zhao J, et al. Mitogenic influence of human Rspondin1 on the intestinal epithelium. Science 2005;309:1256-1259.

42. Chang $L$, Karin M. Mammalian MAP kinase signalling cascades. Nature 2001;410:37-40

43. Cargnello M, Roux PP. Activation and function of the MAPKs and their substrates, the MAPK-activated protein kinases. Microbiol Mol Biol Rev 2011;75:50-83.

44. Jiang $\mathrm{H}$, Edgar BA. EGFR signaling regulates the proliferation of Drosophila adult midgut progenitors. Development 2009;136:483-493.

45. Suzuki A, Sekiya S, Gunshima E, et al. EGF signaling activates proliferation and blocks apoptosis of mouse and human intestinal stem/progenitor cells in long-term monolayer cell culture. Lab Invest 2010;90:1425-1436. 